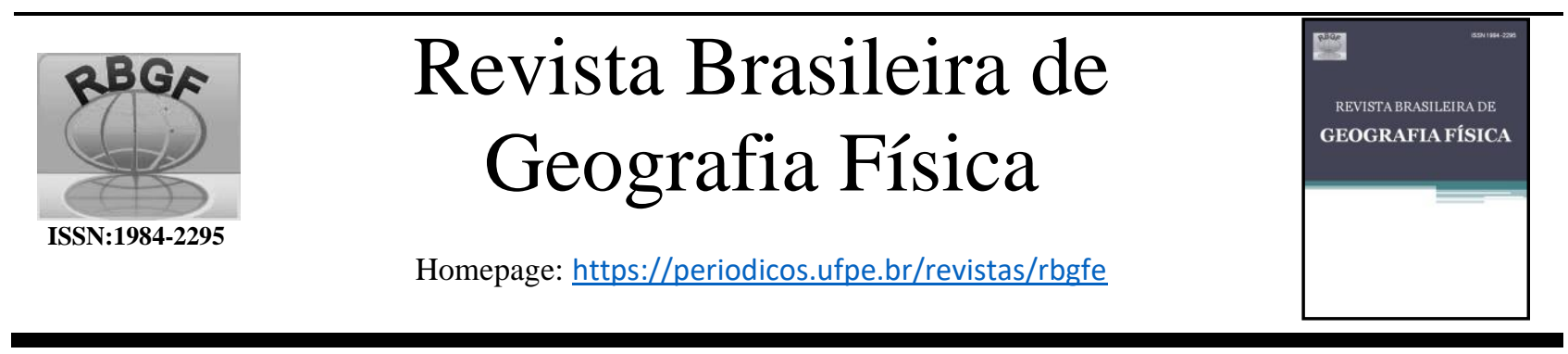

\title{
Análise do Eixo Vertical de dois Ciclones Extratropicais na América do Sul
}

\author{
Lanzoerques Gomes da Silva Júnior ${ }^{1}$, André Becker Nunes ${ }^{2}$
}

${ }^{1}$ Doutorando em Ciências Climáticas, Programa de Pós-Graduação em Ciências Climáticas, Universidade Federal do Rio Grande do Norte, Avenida Senador Salgado Filho, 3000, CEP 59078-970, Lagoa Nova, Natal, Rio Grande do Norte-RN, e-mail: lanzoerques@ gmail.com (autor correspondente). ${ }^{2}$ Prof. Dr. do Programa de Pós-graduação em Meteorologia na Universidade Federal de Pelotas (PPGMET/UFPEL), Endereço: Av. Ildefonso Simões Lopes, 2751, Bairro Arco-Íris, Pelotas-RS, CEP 96060-290, e-mail: beckernunes@gmail.com.

Artigo submetido em 29/04/2020 e aceite 04/02/2021

\section{R E S U M O}

Ciclones extratropicais (CE) são sistemas de baixa pressão que ocorrem em latitudes médias (LM) ao longo do ano. São fenômenos vastamente estudados por serem normalmente associados à eventos de precipitação intensa, tempestades e grandes variações de temperatura. Os CEs são inicialmente identificados em superfície, contudo, também são observados até a tropopausa. Este trabalho analisa a estrutura vertical do ciclo de vida de dois CE formados em dezembro de 2012 e junho de 2014. Foram utilizados dados em ponto de grade $\left(0,5^{\circ} \mathrm{x} 0,5^{\circ}\right)$ do Climate Forecast System Version $2 /$ National Center for Atmospheric Research (CFSv2/NCAR) para identificação e obtenção da defasagem longitudinal dos centros de pressão em superfície e em altos níveis. Ambos os casos apresentaram: maior defasagem (diferença longitudinal entre o sistema em altos e baixos níveis devido à inclinação do seu eixo vertical para oeste) ao longo de seu desenvolvimento, similaridade no período de ocorrência e no menor valor da pressão central, assim como no aprofundamento do ciclone sendo superior a $24 \mathrm{hPa}$ em 24 horas. No inverno (verão) o ciclone obteve maior (menor) defasagem e intensificou-se menos (mais), não sugerindo relação entre a inclinação do eixo vertical e a intensificação do ciclone. A inclinação na vertical para oeste foi mais (menos) acentuada no caso do inverno (verão), além de esboçar um comportamento menos (mais) retilíneo até ficar ocluso. Em ambos os casos a vorticidade ciclônica mais intensa esteve ligeiramente à leste do eixo vertical, estando mais próxima do eixo no caso do verão.

Palavras-chave: instabilidade baroclínica; eixo do cavado; inclinação vertical.

\section{Analysis of Vertical Axis of two Extratropical Cyclones in South America}

\begin{abstract}
A B S T R A C T
Extratropical cyclones (EC) are low pressure systems that occur in mid latitudes (ML) throughout the year. CEs are widely studied phenomena since they are usually associated to events of intense precipitation, storms and large variations of temperature. These systems are initially identified on surface, however, they are also observed up to tropopause. This work analyzes the vertical structure of the life cycle of two EC formed on December 2012 and June 2014. Grid point data $\left(0.5^{\circ} \times 0.5^{\circ}\right)$ from the Climate Forecast System Version 2 / National Center for Atmospheric Research (CFSv2 / NCAR) were used to identify and obtain the longitudinal lag of the pressure centers on the surface and in high levels. Both cases presented: greater lag - longitudinal difference between upper and lower levels due to westward vertical tilt of the axis system - during their development, similarity in the period of occurrence and in the lowest value of central pressure, as well as in the deepening of the cyclone being greater than $24 \mathrm{hPa}$ in 24 hours. In winter (summer) the cyclone obtained a larger (smaller) lag and intensified less (more), suggesting no relationship between the inclination of the vertical axis and the cyclone intensification. The westward vertical tilt was more (less) pronounced in winter (summer) case, besides presenting a behavior less (more) rectilinear until it is occluded. In both cases the most intense cyclonic vorticity was slightly east from vertical axis, being closer of the axis in summer case.
\end{abstract}

Keywords: baroclinic instability; axis of the trough; vertical tilt. 


\section{Introdução}

O termo ciclone refere-se a um centro de baixa pressão atmosférica à superfície em relação ao seu entorno, onde a circulação do ar ao redor do centro ocorre no sentido horário no Hemisfério Sul (HS) e anti-horário no Hemisfério Norte (HN). Estes vórtices de escala sinótica, quando formados entre $30^{\circ}$ e $60^{\circ}$ de latitude, latitudes médias (LM), são denominados de extratropicais. Estudos sobre o comportamento de ciclones extratropicais (CE) e fenômenos atmosféricos associados ocorrem desde os primórdios da meteorologia - mais especificamente, desde a Teoria da Frente Polar (Bjerknes, 1919) - especialmente por impactar na vida das pessoas devido às suas diferentes manifestações como chuva, ventos fortes, mudança brusca de temperatura e eventuais tempestades. Regiões mais suscetíveis a ação de CE - como o sul do Brasil - recebem, consequentemente, maior passagem de Sistemas Frontais (SF) e de Sistemas Convectivos de Mesoescala (SCM), que podem acarretar em danos e/ou perdas de bens materiais e impactar na saúde de pessoas e animais, influenciando diretamente a qualidade de vida e a forma como a sociedade se organiza (Grimm, 2009).

O conhecimento cada vez mais detalhado do CE possibilita aos previsores do tempo realizar previsões com maior acurácia. Assim, especialmente sobre LM na América do Sul (AS) e Oceano Atlântico (OA) adjacente, onde há menos pesquisas se comparado ao $\mathrm{HN}$, as análises dos aspectos relacionados ao seu desenvolvimento como área de formação, trajetória, climatologia, intensidade, estrutura vertical e demais características, são fundamentais para sua melhor compreensão. De acordo com Gan e Rao (1991) a ciclogênese é o aparecimento da primeira isóbara fechada em cartas sinóticas de pressão ao nível médio do mar (PNMM), com persistência da isóbara fechada por pelo menos 24 horas. Tais autores realizaram uma climatologia de ciclogênese à superfície para a AS baseando-se em dez anos (1979-1988) de dados de cartas sinóticas e de estações meteorológicas, onde localizaram duas regiões ciclogenéticas preferenciais sobre a costa leste da AS, sendo uma no sudeste da Argentina e outra no Uruguai. Tais resultados foram confirmados por Reboita et al. (2010) por meio de climatologia de dados de vorticidade relativa para o período de 1990 a 1999.
Os CE são, tipicamente, sistemas baroclínicos. Conforme os trabalhos de Charney (1947) e Eady (1949), a instabilidade baroclínica é função do gradiente meridional de temperatura (característico de LM) e, consequentemente, do cisalhamento vertical, apresentando inclinação do seu eixo para oeste com a altura (Figura 1). Esta inclinação vertical para oeste, sobre a massa fria (Reboita et al., 2017a), indica que o sistema está convertendo energia, i.e., em desenvolvimento.

$\mathrm{Na}$ consequente evolução do ciclo de vida, o sistema em altos níveis se desloca mais rapidamente que o de baixos níveis, sobrepondo-o, na chamada fase de oclusão barotrópica (Palmén e Newton, 1969; Bluestein, 1993). A região de maior baroclinia está relacionada com a trajetória das tempestades, sendo esta a região preferencial de deslocamento dos $\mathrm{CE}$, podendo ser identificada onde se encontra a máxima energia cinética dos sistemas transientes, tendo como característica fortes gradientes de variáveis como geopotencial e vento meridional (Rao et al., 2002).

Como sistemas baroclínicos, $\mathrm{CE}$ convertem energia potencial do estado básico (EPD) em energia potencial da perturbação (EPD') devido à sua inclinação vertical sobre o lado frio (oeste), a qual está associada ao gradiente meridional de temperatura. Tal inclinação diminui com o desenvolvimento baroclínico - cuja variação com a altura não necessariamente apresenta eixo de formato linear - até a fase de conversão de EPD' em energia cinética da perturbação (K'), finalizando o ciclo básico de energia de um sistema baroclinicamente instável (Brian, 1982).

A literatura é vasta quanto ao estudo de desenvolvimento e evolução do ciclo de vida de CE no HS, assim como AS e AO especificamente (Taljaard, 1967; Satyamurty et al., 1990; Gan e Rao, 1991; Sinclair, 1994; Mendes et al., 2007; 2010; Reboita et al., 2017a; Schultz et al., 2018), mas ainda são necessários mais trabalhos quanto à análise de fatores que influenciam nas diferentes estruturas verticais observadas. A estrutura vertical do ciclone pode indicar sua natureza (baroclínico ou barotrópico), fase de desenvolvimento e até comportamento da precipitação (Pepler e Dowdy, 2020). Assim, o objetivo deste trabalho é comparar o desenvolvimento baroclínico - especificamente com relação à inclinação vertical do eixo do cavado - de dois casos de CE que se formaram sobre a AS em diferentes estações do ano (verão e inverno), visando aprofundar o conhecimento do ciclo de vida destes sistemas na América do Sul. Os casos 
em questão ocorreram em 31 de dezembro de 2012 e 12 de junho de 2014.

\section{Material e métodos}

Utilizou-se dados em pontos de grade com resolução espacial de $0,5^{\circ}$ de latitude e $0,5^{\circ}$ de longitude, do Climate Forecast System Version 2/National Center for Atmospheric Research (CFSv2/NCAR) (Saha et al., 2014), em intervalos de tempo de seis horas. As variáveis meteorológicas utilizadas para acompanhar o desenvolvimento dos CE foram PNMM, altura geopotencial e componentes zonal (u) e meridional (v) do vento em vários níveis verticais para obtenção do campo de vorticidade. O domínio espacial abrangeu o continente sul-americano e oceanos adjacentes.

Também foram utilizadas imagens de satélite do canal de vapor d'água do Geostationary Operational Environmental Satellites (GOES) 13, por meio do banco de dados de imagens da Divisão de Satélites de Sistemas Ambientais (DSA), do Instituto Nacional de Pesquisas Espaciais (INPE), disponíveis em

(http://satelite.cptec.inpe.br/home/novoSite/index. jsp).

Aqui, espera-se obter eixos verticais como o esquema teórico mostrado em Holton (2004), onde se observa a inclinação do eixo do cavado ao longo da troposfera (Figura 1). Essa característica está relacionada ao deslocamento para oeste dos centros de pressão à medida que se analisa níveis báricos mais elevados.

A fase de ciclogênese é definida por meio de uma isóbara que se mantém fechada por pelo menos 24 horas (Gan e Rao, 1991). Após o período de ciclogênese o CE se desenvolve até o estágio de oclusão (sem inclinação vertical), podendo também alcançar a fase de dissipação, sendo esta a última etapa do seu ciclo de vida.

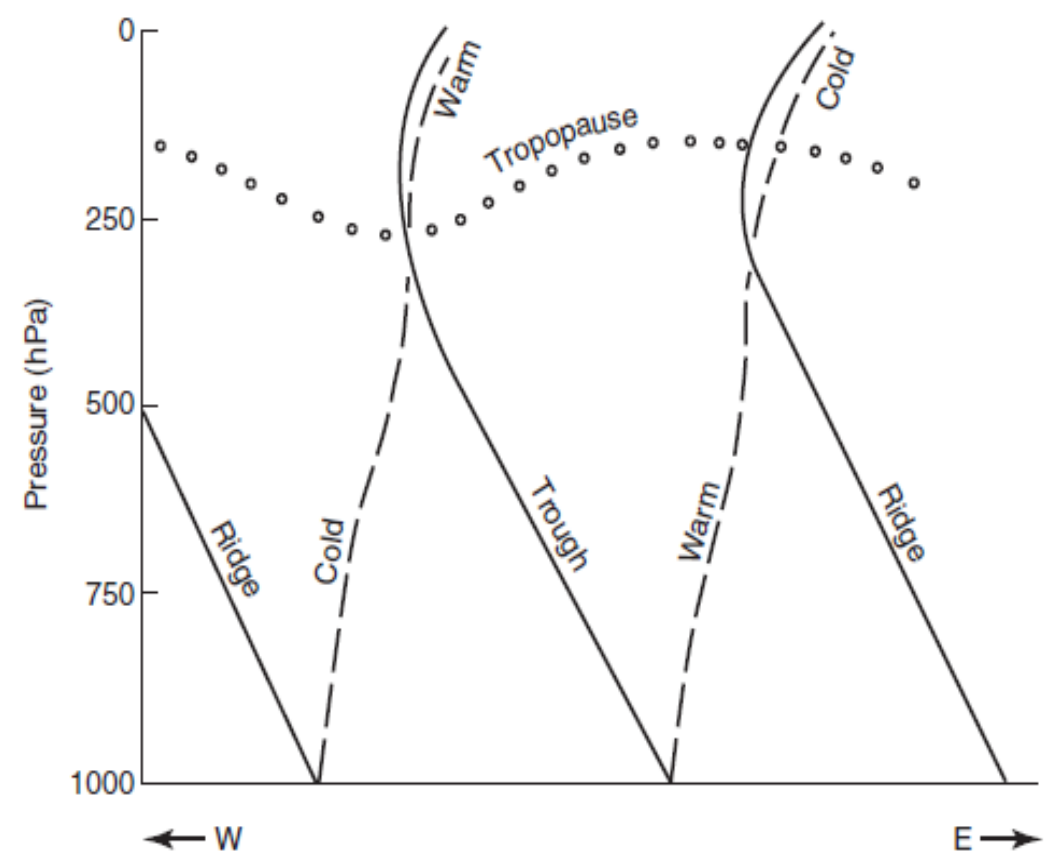

Figura 1. Seção transversal oeste-leste de uma onda baroclínica em desenvolvimento. Linhas sólidas são eixos de crista e cavado; linhas tracejadas são eixos de temperatura extrema; a linha de círculos abertos denota a tropopausa.

Fonte: Holton, 2004.

A defasagem, em graus de longitude, entre o sistema em superfície e o de altos níveis (250 $\mathrm{hPa}$ ) foi obtida por meio da diferença entre as longitudes dos centros de baixa pressão destes sistemas. No sistema em superfície o centro foi determinado pela isóbara fechada de menor valor enquanto que para os altos níveis o eixo do cavado foi observado com base na componente meridional (v) do vento tendendo a zero, ou seja, valores positivos seguidos de negativos indicam eixos de centro de baixa pressão. Em altos níveis, o sistema de baixa pressão é observado quando da presença 
de cavado ou, nos casos mais intensos, por vórtices ciclônicos de altos níveis do tipo Palmén (Palmén e Newton, 1969; Nunes, 2017).

\section{Resultados e discussão}

Por meio de imagens de satélite, campos horizontais e seções verticais, pôde-se acompanhar o ciclo de vida dos ciclones em superfície e o comportamento do cavado em altos níveis, analisando-os desde sua formação até o estágio de oclusão. Observando-se as Figuras 2 e 3, verão e inverno respectivamente, nota-se pela altura geopotencial em $250 \mathrm{hPa}$ (linhas tracejadas) que para ambos os casos a região corrente abaixo do cavado em altos níveis - ou seja, região de advecção de vorticidade ciclônica (AVC) encontra-se sobre a área do ciclone em superfície (campo de PNMM representado por isolinhas pretas contínuas, a seta verde indica o centro do ciclone em análise), concordando com a literatura sobre a formação de CE (Palmén e Newton, 1969). Ambos casos tiveram formação sobre a Argentina, sendo o caso de verão próximo à zona costeira $\left(40,5^{\circ} \mathrm{S}\right.$ e $\left.60^{\circ} \mathrm{W}\right)$ e inverno na região central $\left(32^{\circ} \mathrm{S}\right.$ e $68^{\circ} \mathrm{W}$ ). Em um passo anterior à formação dos ciclones (não mostrado aqui) notou-se que, em baixos níveis, ambos casos foram originados de cavados térmicos continentais - sejam eles associados à Baixa do Chaco (Lenters e Cook, 1999) no caso do verão ou Baixa do Noroeste Argentino (Seluchi et al., 2003) no caso do inverno.
É comum a formação de ciclones extratropicais na costa do Uruguai originados de sistemas de baixa pressão continentais em baixos níveis (Caballero et al., 2018; Riquetti et al., 2018; Dornelles et al., 2020).

O campo sombreado em azul e vermelho, ventos de sul e de norte respectivamente, indica o eixo dos centros de pressão no nível de $250 \mathrm{hPa}$.

Assim, para as $12 \mathrm{Z}$ de 31/12/2012 o eixo do cavado em altos níveis encontrava-se em $65^{\circ} \mathrm{W}$ e o centro do ciclone em superfície em $60^{\circ} \mathrm{W}$, apresentando uma diferença aproximada de $5^{\circ}$. O caso do inverno apontou maior defasagem inicial, $9^{\circ}$ às $00 \mathrm{Z}$ de $12 / 06 / 2014$, com o centro do cavado em $77^{\circ} \mathrm{W}$ e do ciclone em $68^{\circ} \mathrm{W}$.

Outro aspecto a se destacar é o eixo horizontal do cavado em altos níveis. No caso do verão nota-se, durante a fase de desenvolvimento, uma inclinação positiva (MacDonald, 1976). Esta inclinação horizontal segue diminuindo à medida que se aproximava do estágio de oclusão. Trabalhos no $\mathrm{HN}$ indicam maior probabilidade de instabilidade severa em casos de inclinação negativa devido a maior velocidade vertical ascendente (Glickman et al., 1977), mas tal comportamento não é tão facilmente observado em eventos na AS (Cossetin et al., 2016). Também é comum se observar a variação da inclinação ao longo do desenvolvimento do ciclone, conforme observado aqui. Já no caso de inverno, a inclinação horizontal não se apresenta muito clara. 

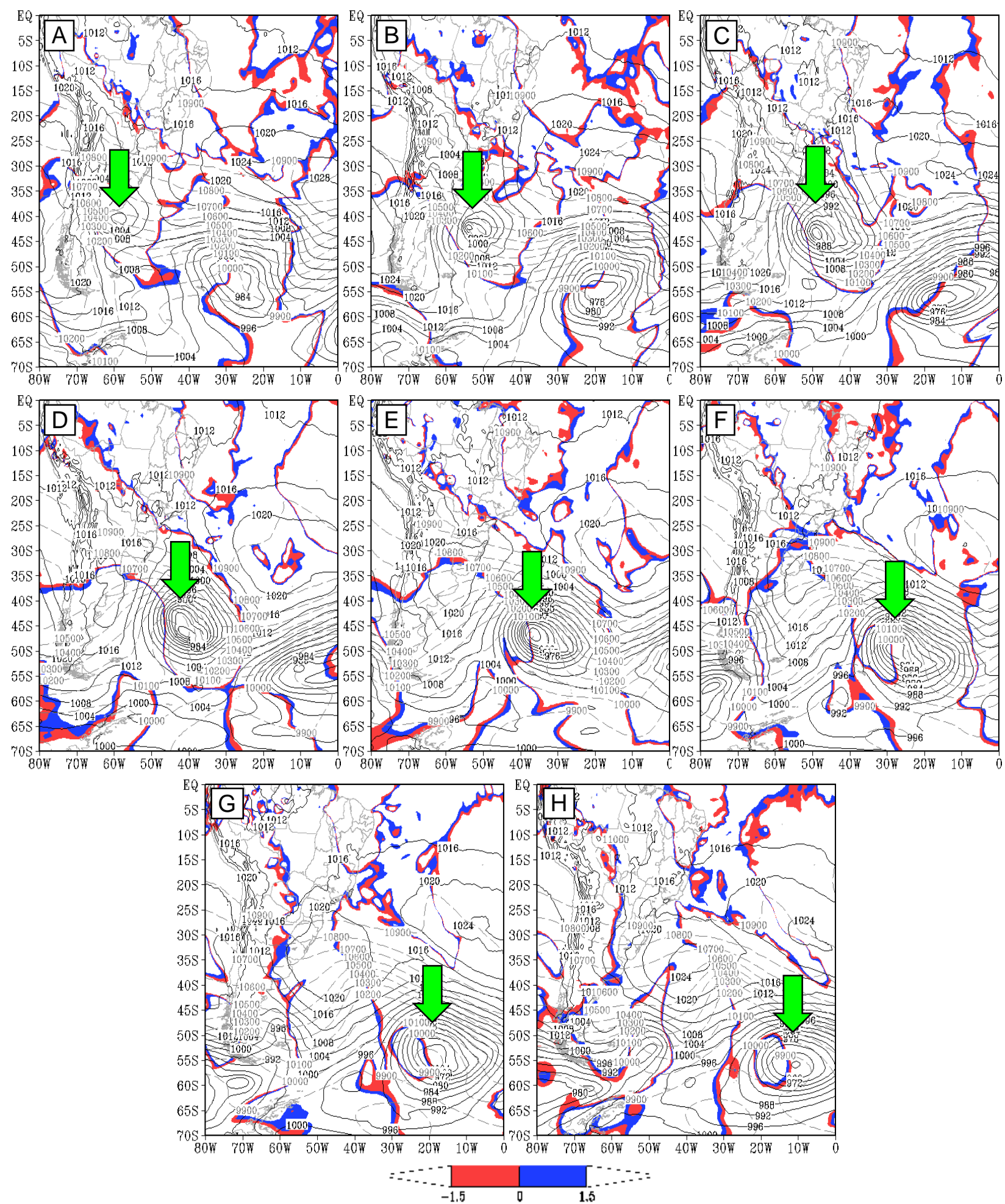

Figura 2. Campo de PNMM (contorno contínuo cinza, em hPa), altura geopotencial em $250 \mathrm{hPa}$ (contorno tracejado preto, em mgp) e componente meridional do vento (sombreado, em m s${ }^{-1}$ ) em $250 \mathrm{hPa}$. (A) 31/12/12 12Z, (B) 01/01/13 00Z, (C) 01/01/13 12Z, (D) 02/01/13 00Z, (E) 02/01/13 12Z, (F) 03/01/13 00Z, (G) 03/01/13 12Z, (H) 04/01/13 00Z. 

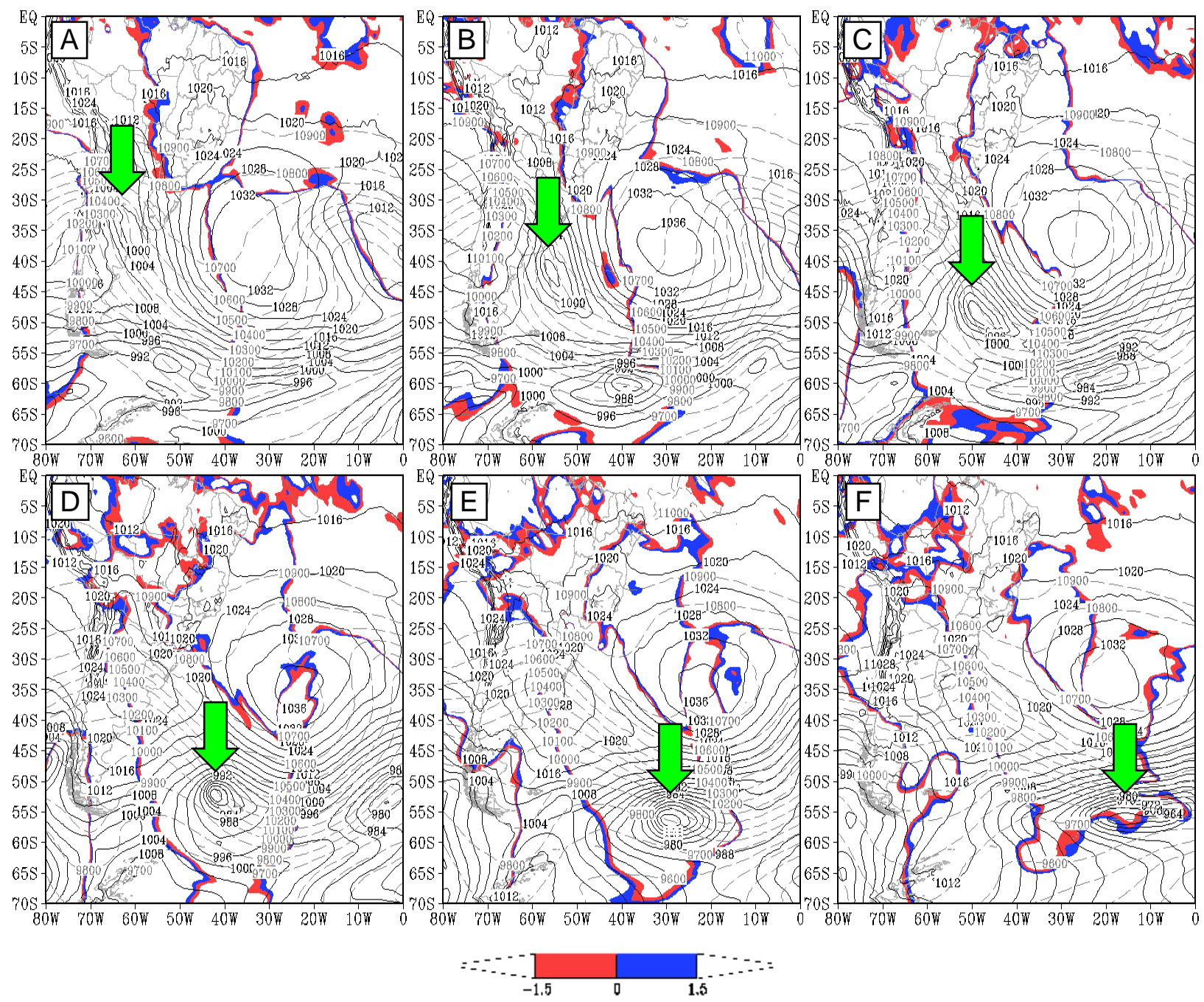

Figura 3. Campo de PNMM (contorno contínuo cinza, em hPa), altura geopotencial em $250 \mathrm{hPa}$ (contorno tracejado preto, em mgp) e componente meridional do vento (sombreado, em m s${ }^{-1}$ ) em $250 \mathrm{hPa}$. (A) 12/06/14 12Z, (B) 13/06/14 00Z, (C) 13/06/14 12Z, (D) 14/06/14 00Z, (E) 14/06/14 12Z, (F) 15/06/14 00Z.

As Figuras 4 e 5 são imagens de satélite do canal de vapor d'água que evidenciam a banda de nebulosidade formada pelo avanço do SF - e consequente nebulosidade devido à advecção quente - do CE. Nos dois casos é possível observar um setor relativamente mais seco após a passagem do SF, sugerindo a presença de ar frio proveniente da baixa estratosfera, típico de ciclones intensos com rebaixamento da tropopausa dinâmica
(Vaughan et al., 1994; Santurette e Georgiev, 2005; Avila et al., 2016; Nunes e Avila, 2017).

Esse contraste de banda de nebulosidade com setor mais seco é mais facilmente observado no canal de vapor d'água devido ao alcance da intrusão de ar estratosférico, contribuindo para a identificação dos sistemas devido aos contornos que eles apresentam. 
Revista Brasileira de Geografia Física v.14, n.01 (2021) 068-081.
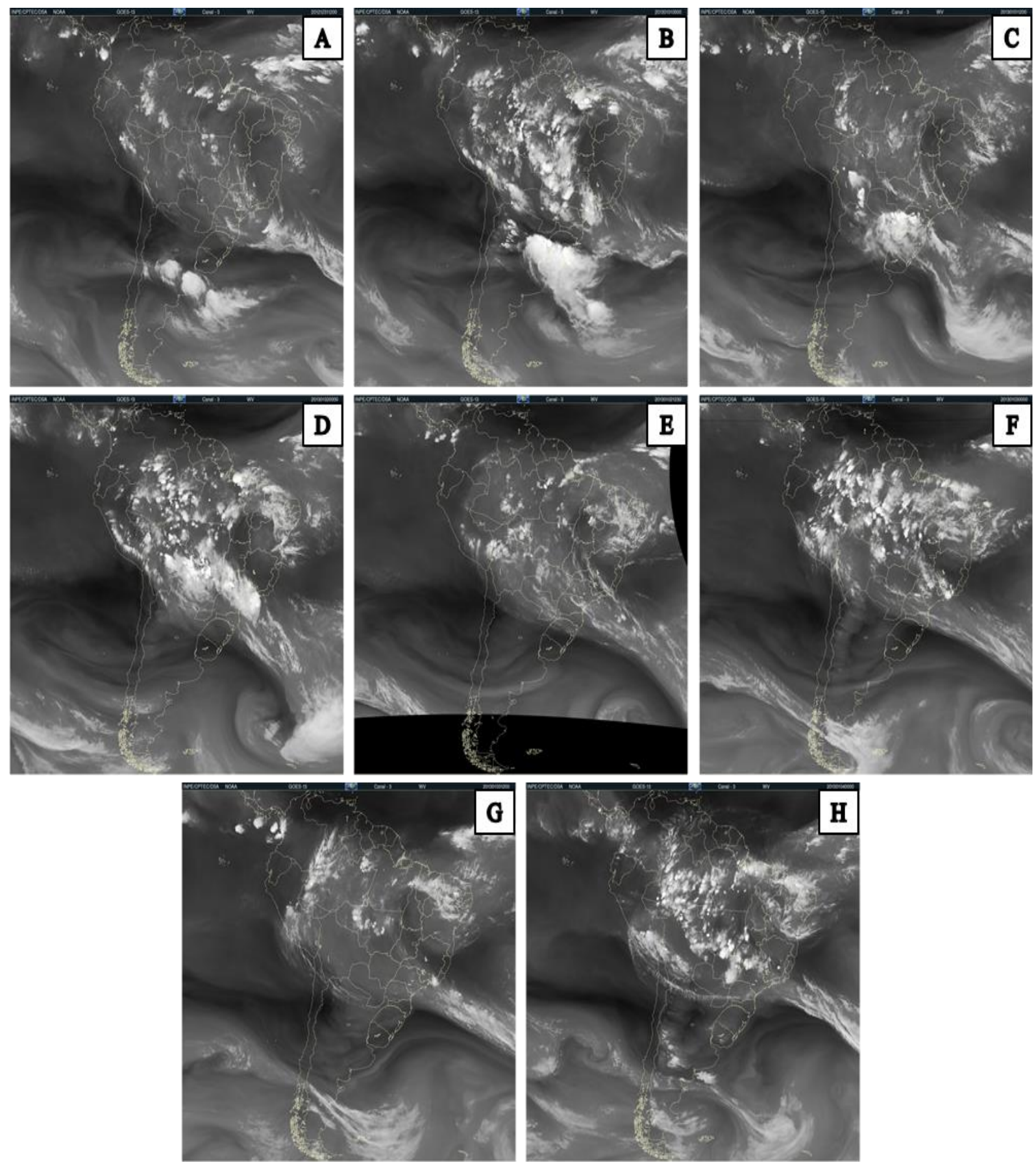

Figura 4. Imagens de satélite do canal de vapor d'água. (A) 31/12/12 12Z, (B) 01/01/13 00Z, (C) 01/01/13 12Z, (D) 02/01/13 00Z, (E) 02/01/13 12Z, (F) 03/01/13 00Z, (G) 03/01/13 12Z, (H) 04/01/13 00Z. 

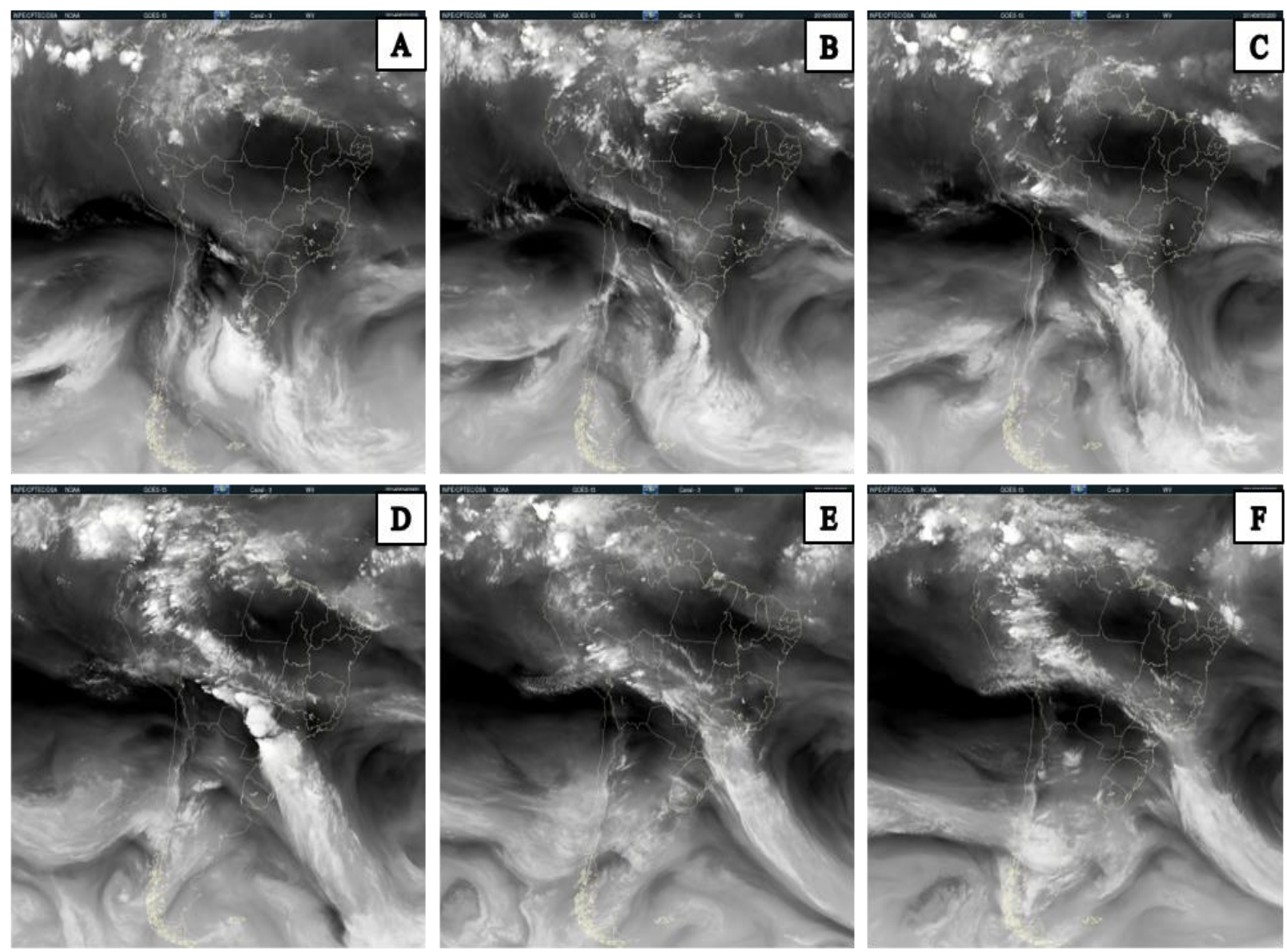

Figura 5. Imagens de satélite do canal de vapor d'água. (A) 12/06/14 12Z, (B) 13/06/14 00Z, (C) 13/06/14 12Z, (D) 14/06/14 00Z, (E) 14/06/14 12Z, (F) 15/06/14 00Z.

O campo da estrutura vertical dos sistemas, Figuras 6 e 7, evidenciou a inclinação do eixo para oeste juntamente com a seção vertical da vorticidade relativa ciclônica, característica de sistemas baroclínicos, indicando que o sistema ainda está convertendo EPD em EPD'. Nos intervalos seguintes a inclinação vertical do eixo e da vorticidade diminuem, o que configura o início da fase de oclusão do sistema, em conformidade com as Figuras 2 e 3. Ressalta-se, aqui, a diferença entre a inclinação vertical para oeste teórica (Figura 1) e as apresentadas neste trabalho, baseadas em dados em ponto de grade. Tal diferença se deve ao fato de que a teoria deve esquematizar os fenômenos de forma clara e simples para que seja didática, enquanto que as estimativas tentam apresentar os fenômenos da forma mais realista possível.

Os dois sistemas apresentaram à sotavento de seu eixo vertical a ação da vorticidade relativa ciclônica, que no caso do verão (inverno) se apresentou um pouco mais próxima (distante) ao eixo. Ambos casos obtiveram dois máximos de vorticidade ao longo da estrutura vertical, sendo em torno de $900 \mathrm{hPa}$ e $400 \mathrm{hPa}$ no verão, e próximo à $900 \mathrm{hPa}$ e $300 \mathrm{hPa}$ no inverno. Resultados similares foram encontrados em estudo de caso de maio de 2013 por Reboita et al. (2017b). O caso de inverno mostrou uma estrutura vertical mais sinuosa e com vorticidade atuando de forma descontínua ao longo de sua extensão vertical nas primeiras 24 horas. De modo contrário, no verão o eixo esteve um pouco menos curvo e com vorticidade atuando em toda a seção vertical. Em análise de três casos no Atlântico Sul, Abreu et al. (2018) também verificou diferentes comportamentos verticais do campo de vorticidade relativa ao longo dos ciclones de vida dos ciclones. 

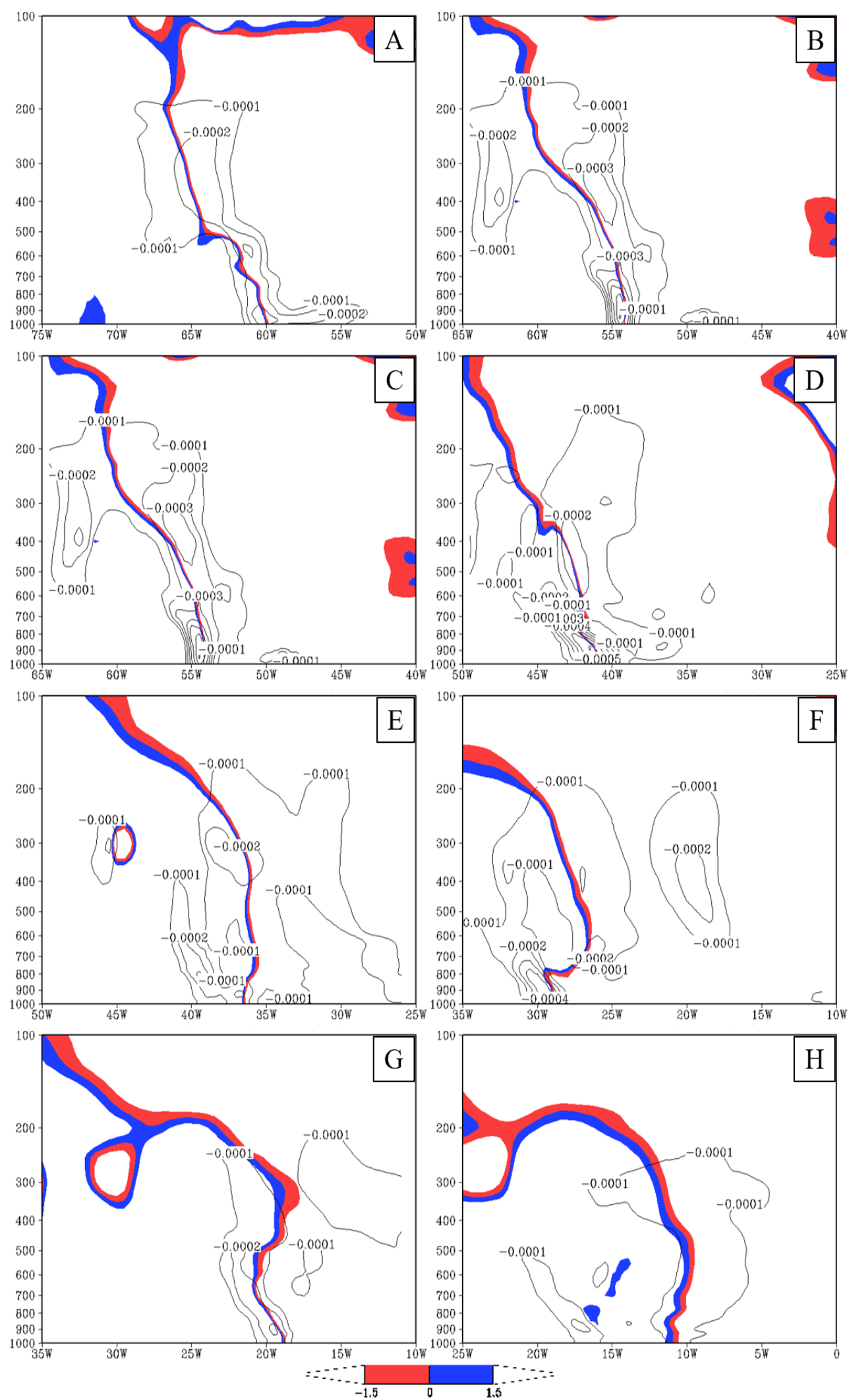

Figura 6. Campo de componente meridional do vento (sombreado, em $\mathrm{m} \mathrm{s}^{-1}$ ) e vorticidade relativa (contorno pontilhado preto, $\mathrm{em} \mathrm{s}^{-1}$ ). (A) 31/12/12 $12 \mathrm{Z}$ (lat. -40,5), (B) 01/01/13 00Z (lat. -42), (C) 01/01/13 12Z (lat. 43,5), (D) 02/01/13 00Z (lat. -46,5), (E) 02/01/13 12Z (lat. -47), (F) 03/01/13 00Z (lat. -50), (G) 03/01/13 $12 \mathrm{Z}$ (lat. -54,5), (H) 04/01/13 00Z (lat. -55,5). 

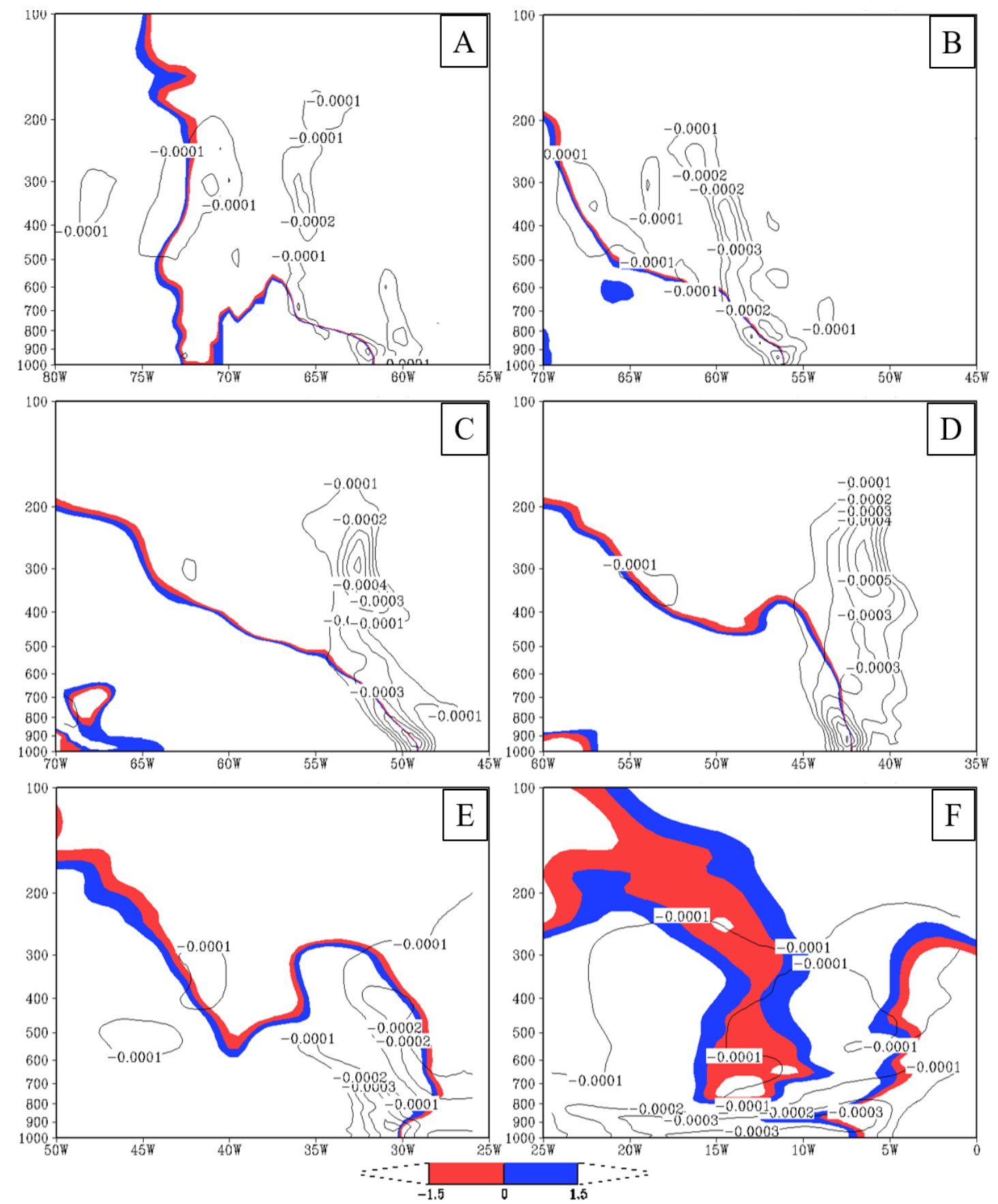

Figura 7. Campo de componente meridional do vento (sombreado, $\mathrm{em} \mathrm{m} \mathrm{s}^{-1}$ ) e vorticidade relativa (contorno pontilhado preto, $\mathrm{em} \mathrm{s}^{-1}$ ). (A) 12/06/14 $12 \mathrm{Z}$ (lat. -35,5), (B) 13/06/14 00Z (lat. -41,5), (C) 13/06/14 12Z (lat. 48,5), (D) 14/06/14 00Z (lat. -52,5), (E) 14/06/14 12Z (lat. -56,5), (F) 15/06/14 00Z (lat. -56,5).

O tempo total do ciclo de vida dos ciclones foi considerado desde a formação do centro de baixa pressão em superfície até o instante em que deixa de apresentar inclinação com a vertical (defasagem de $0^{\circ}$ ), sendo os sistemas observados até o limite do domínio espacial situado no Meridiano de Greenwich. Conforme apresentado pela Tabela 1 o caso de verão teve duração de três dias e 18 horas, com a fase barotrópica ocorrendo após dois dias e 12 horas, 12 horas a mais que no caso de inverno (Tabela 2), o qual só atingiu a fase barotrópica no último passo de tempo observado.

Para ambos os casos a maior defasagem não ocorreu durante a fase de ciclogênese, mas sim ao longo de seu desenvolvimento, sendo de $7^{\circ}$ (verão) e $17,5^{\circ}$ (inverno). Nota-se que, de modo geral, ao longo do ciclo de vida dos ciclones, o caso de verão (inverno) apresentou inclinação menos 
(mais) acentuada, em média $3^{\circ}\left(12^{\circ}\right)$. Ambos os casos apresentaram aprofundamento da pressão central superior a $24 \mathrm{hPa}$ em 24 horas, como pode ser observado das $6 \mathrm{Z}$ de $01 / 01 / 13$ às $6 \mathrm{Z}$ de $02 / 01 / 13(25 \mathrm{hPa})$, e em $12 \mathrm{Z}$ de 13/06/14 às $12 \mathrm{Z}$ de 14/06/14 (27,2 hPa), característica de ciclone explosivo, conforme Sanders e Gyakum (1980). Observou-se, também em ambos casos, que a defasagem não decaiu linearmente a cada passo de tempo; ao longo do ciclo de vida o decaimento da defasagem apresentou intervalos

com retrocessos, que não apresentaram relação direta com os retrocessos obervados no decaimento da PNMM.

Outras duas similaridades apresentadas pelos dois ciclones referem-se ao menor valor obtido da pressão central e ao período de sua ocorrência, sendo 12 horas antes de atingir a fase barotrópica, onde, para o caso de verão (inverno), a pressão foi de $956,3 \mathrm{hPa}(957,2 \mathrm{hPa})$ às $6 \mathrm{Z}$ de $02 / 01 / 13$ (às $12 Z$ de 14/06/14). Isso ocorre porque a energia cinética produzida pelo ciclone é usada em parte para intensificar o seu aprofundamento. Sabe-se que o ciclone obtém a maior intensidade durante o processo de oclusão, quando o setor quente é forçado a subir, rebaixando o centro de gravidade do sistema, convertendo energia potencial da massa em energia cinética da perturbação, fazendo com que essa fase apresente as maiores quedas de pressão à superfície (Peixoto e Oort, 1992; Mathias, 2012).

Tabela 1 Intensificação do ciclone (hPa) e defasagem (graus de longitude) com o nível de 250 hPa no caso do verão.

\begin{tabular}{cccccc}
\hline Horas & $31 / 12 / 2012$ & $01 / 01 / 2013$ & $02 / 01 / 2013$ & $03 / 01 / 2013$ & $04 / 01 / 2013$ \\
\hline 0 & ----- & $985,8-6^{\circ}$ & $965,7-6^{\circ}$ & $961,6-\left(-0,5^{\circ}\right)$ & $963,6-2^{\circ}$ \\
6 & ---- & $981,3-5^{\circ}$ & $956,3-1,5^{\circ}$ & $960,9-0^{\circ}$ & ---- \\
12 & $997,1-5^{\circ}$ & $977-3^{\circ}$ & $958,1-1^{\circ}$ & $960,6-1,5^{\circ}$ & ---- \\
18 & $990,7-7^{\circ}$ & $972,3-3^{\circ}$ & $960,5-\left(-0,5^{\circ}\right)$ & $963,1-2^{\circ}$ & ---- \\
\hline
\end{tabular}

Tabela 2 Intensificação do ciclone (hPa) e defasagem (graus de longitude) com o nível de 250 hPa no caso do inverno.

\begin{tabular}{ccccc}
\hline Horas & $12 / 06 / 2014$ & $13 / 06 / 2014$ & $14 / 06 / 2014$ & $15 / 06 / 2014$ \\
\hline 0 & $997-9^{\circ}$ & $993,6-14^{\circ}$ & $968,3-14^{\circ}$ & $961,7-\left(-4^{\circ}\right)$ \\
6 & $998-10,5^{\circ}$ & $989-17,5^{\circ}$ & $963,7-17^{\circ}$ & ----- \\
12 & $997,5-11,5^{\circ}$ & $984,4-15,5^{\circ}$ & $957,2-14^{\circ}$ & ----- \\
18 & $993,7-13^{\circ}$ & $979-17^{\circ}$ & $959,5-1,5^{\circ}$ & ----- \\
\hline
\end{tabular}

Comparando-se as características de cada caso, no inverno (verão) o ciclone apresentou maior (menor) defasagem, intensificou-se menos (mais) e mais devagar (rápido). Dessa forma não se verificou relação direta entre a taxa de aprofundamento da pressão central do ciclone com a inclinação vertical do sistema.

\section{Conclusões}

Este estudo analisou o comportamento da estrutura vertical de dois casos de CE ocorridos nos dias 31 de dezembro de 2012 e 12 de junho de 2014. Foi possível determinar a distância (defasagem) em graus de longitude do centro de baixa pressão na superfície e em $250 \mathrm{hPa}$, para posteriormente se comparar o comportamento da defasagem ao longo da evolução do sistema, assim como se analisar a inclinação do eixo na seção vertical. Embora os casos sejam de diferentes estações do ano eles demonstraram bastante similaridade com relação à trajetória realizada, inclinação durante a fase de desenvolvimento, instante e valor de menor pressão central, assim como o estágio de oclusão 12 horas após a maior intensidade.

A inclinação vertical para oeste se apresentou mais (menos) acentuada no caso do inverno (verão), além de esboçar um comportamento menos (mais) retilíneo na vertical até chegar à fase de oclusão. Ambos os casos obtiveram valores significativos de vorticidade ciclônica um pouco à leste do eixo vertical, estando mais próximo do eixo no caso do verão.

Assim como o decaimento da PNMM (intensificação do ciclone) não foi linear, ou seja, 
durante o ciclo de vida notou-se intervalos com aumento de pressão, o mesmo se pode dizer com relação ao decaimento da defasagem, embora não se tenha observado relação direta entre a variação temporal da defasagem e da queda da PNMM.

Para aprofundar o conhecimento sobre o comportamento dos CE é necessário analisar um maior número de casos, relacionando a intensificação do sistema de baixa pressão na superfície com a inclinação para oeste na vertical.

\section{Agradecimentos}

O primeiro autor agradece ao Conselho Nacional de Desenvolvimento Científico e Tecnológico $(\mathrm{CNPq})$ pela concessão de bolsa de mestrado.

\section{Referências}

Abreu, E. X., Silva, M. V., Reboita, M. S., Teodoro, T. A., 2018. Study of the life cycle of three extratropical cyclones in the South Atlantic Ocean. Revista Brasileira de Geografia Física [Online] 11. Disponível: https://doi.org/10.26848/rbgf.v10.6.p251275. Acesso: 15 abr. 2020.

Avila, V.D., Nunes, A.B., Alves, R.C.M., 2016. Analysis of an explosive cyclogenesis case occurred in 03/01/2014 on the south of Atlantic Ocean. Revista Brasileira de Geografia Física [Online] 9. Disponível: https://doi.org/10.26848/rbgf.v9.4.p10881099. Acesso: 15 abr. 2020.

Bjerknes, J., 1919. On the structure of moving cyclones. Monthly Weather Review [Online] 47. Disponível: https://doi.org/10.1175/15200493(1919)47<95:OTSOMC >2.0.CO;2. Acesso: 15 abr. 2020.

Bjerknes, J., Solberg, H., 1922. Life cycle of cyclones and the polar front theory of atmospheric circulation. Geofysiske Publikasjoner 3, 3-18.

Brian, F.F., 1982. The initial growth of disturbances in a baroclinic flow. Journal of the Atmospheric Sciences [Online] 39. Disponível: https://doi.org/10.1175/15200469(1982)039<1663:TIGODI>2.0.CO;2.

Acesso: 15 abr. 2020.

Bluestein, H.B., 1993. Synoptic-Dynamic Meteorology in Midlatitudes. Volume II: Observations and theory of weather systems, Oxford University Press, Oxford.
Caballero, C. B., Ogassawara, J. F., Dorneles, V. R., Nunes, A. B. 2018. A precipitação pluviométrica em Pelotas/RS: Tendência, sistemas sinóticos associados e influência da ODP. Revista Brasileira de Geografia Física [Online] 11. Disponível: https://doi.org/10.26848/rbgf.v11.4.p14291441. Acesso: 15 abr. 2020.

Charney, J. G., 1947. The dynamics of long waves in a baroclinic westerly current. Journal of Meteorology [Online] 4. Disponível: https://doi.org/10.1175/15200469(1947)004<0136:TDOLWI>2.0.CO;2. Acesso: 15 abr. 2020.

Dorneles, V. R., Riquetti, N. B., Nunes, A. B., 2020. Forçantes dinâmicas e térmicas associadas a um caso de precipitação intensa sobre o Rio Grande do Sul, Brasil. Revista Brasileira de Climatologia [Online] 26. Disponível: http://dx.doi.org/10.5380/abclima.v26i0.5816 2. Acesso: 15 abr. 2020.

Cossetin, F., Nunes, A. B., Teixeira, M. S., 2016. Análise do movimento vertical sob duas diferentes configurações de altos níveis da troposfera. Ciência e Natura [Online] 38. Disponível: http://dx.doi.org/10.5902/2179460X20281. Acesso: 15 abr. 2020.

Eady, E. T., 1949. Long waves and cyclone waves. Tellus [Online] 1. Disponível: https://doi.org/10.3402/tellusa.v1i3.8507. Acesso: 15 abr. 2020.

Gan, M.A., Rao, V.B., 1991. Surface cyclogenesis over South America. Monthly Weather Review [Online] 119. Disponível: https://doi.org/10.1175/15200493(1991)119<1293:SCOSA >2.0.CO;2. Acesso: 15 abr. 2020.

Glickman, T., MacDonald, N. J., Sanders, F., 1977. New findings on the apparent relationship between convective activity and the shape of $50 \mathrm{mb}$ troughs. Monthly weather Review [Online] $105 . \quad$ Disponível: https://doi.org/10.1175/15200493(1977)105<1060:NFATAR>2.0.CO;2. Acesso: 15 abr. 2020.

Grimm, M.A., 2009. Clima da Região Sul do Brasil, in: Cavalcanti, I.F.A., Ferreira, N.J., Silva, M.G.A.J, Silva Dias, M.A.F., (Org), Tempo e Clima no Brasil. 1 ed. Oficina de Textos, São Paulo, 259- 275.

Holton, J.R., 2004. An Introduction to Dynamic Meteorology, Elsevier, San Diego. 
Lenters, J. D., Cook, K. H., 1999. Summertime precipitation variability over South America: Role of the large-scale circulation. Monthly Weather Review [Online] 127. Disponível: https://doi.org/10.1175/15200493(1999)127<0409:SPVOSA>2.0.CO;2.

Acesso: 16 abr. 2020.

MacDonald, N. J., 1976. On the apparent relationship between convective activity and the shape of $500 \mathrm{mb}$ troughs. Monthly Weather Review [Online] 104. Disponível: https://doi.org/10.1175/15200493(1976)104<1618:OTARBC>2.0.CO;2. Acesso: 16 abr. 2020.

Mathias, R. S. B., 2012. Análise e Classificação de Ciclones Utilizando Diagramas de Fase: Conceitos e Aplicação em Previsão de Tempo Operacional. Dissertação (Mestrado). Rio de Janeiro, UFRJ.

Mendes, D., SOUZA, E. P., TRIGO, I. F., MIRANDA, P. M. A., 2007. On Precursors of South-American Cyclogenesis. Tellus A [Online] 59. Disponível:

https://doi.org/10.1111/j.16000870.2006.00215.x. Acesso: 16 abr. 2020.

Mendes, D., Souza, E.P., Marengo, J.A., Mendes, M.C.D., 2010. Climatology of extratropical cyclones over the South American-southern oceans sector. Theoretical and Applied Climatology [Online] 100. Disponível: https://doi.org/10.1007/s00704-009-0161-6. Acesso: 16 abr. 2020.

Nunes, A.B., Avila, V.D., 2017. Dynamic tropopause analysis: Case Study in South America. Anuário do Instituto de Geociências - UFRJ [Online] 40. Disponível: http://dx.doi.org/10.11137/2017_1_83_92. Acesso: 16 abr. 2020.

Nunes, A.B., 2017. Estudo de caso de sistemas meteorológicos em altos níveis sobre a América do Sul: Análise Sinótica. Anuário do Instituto de Geociências - UFRJ [Online] 40. Disponível:

http://dx.doi.org/10.11137/2017 $1 \quad 70 \quad 82$. Acesso: 16 abrl. 2020.

Palmén, E., Newton, C.W., 1969. Atmospheric Circulation Systems: their Structure and Physical Interpretation, 1 ed. Academic Press, New York.

Peixoto, J. P.; Oort, A. H. 1992. Physics of Climate, 1 ed. American Institute of Physics, New York.

Pepler, A., Dowdy, A., 2020 A three-dimensional perspective on extratropical cyclone impacts.
Journal of Climate [Online] 0. Disponível: https://doi.org/10.1175/JCLI-D-19-0445.1. Acesso: 16 abr. 2020.

Rao, V. B., Carmo, A. M. C., Franchito, S. H., 2002. Seasonal variations in the Southern Hemisphere storm tracks and associated wave propagation. Journal Atmospheric Sciencies [Online] 59.5 Disponível: https://doi.org/10.1175/15200469(2002)059<1029:SVITSH $>2.0 . \mathrm{CO} ; 2$. Acesso: 16 abr. 2020.

Reboita, M. S., Da Rocha, R. P., Ambrizzi, T., Sugahara, S., 2010. South Atlantic Ocean cyclogenesis climatology simulated by regional climate model (RegCM3). Climate Dynamics [Online] 35. Disponível: http://dx.doi.org/10.1007/s00382-009-06687. Acesso: 16 abr. 2020.Reboita, M. S., Gan, M. A., Da Rocha, R. P., Custódio, I. S., 2017a. Ciclones em Superfície nas Latitudes Austrais: Parte I - Revisão Bibliográfica. Revista Brasileira de Meteorologia [Online] 32. Disponível: https://doi.org/10.1590/0102-77863220010. Acesso: 16 abr. 2020.

Reboita, M. S., Gan, M. A., Da Rocha, R. P., Custódio, I. S., 2017b. Ciclones em Superfície nas Latitudes Austrais: Parte II Estudo de Casos. Revista Brasileira de Meteorologia [Online] 32. Disponível: https://doi.org/10.1590/0102-7786324002. Acesso: 16 abr. 2020.

Riquetti, N. B., Dorneles, V. R., Nunes, A. B., 2018. Estudo do caso de precipitação intensa sobre Foz do Iguaçu-PR em setembro de 2015. Revista Brasileira de Climatologia [Online] 22. Disponível:

http://dx.doi.org/10.5380/abclima.v22i0.5830 4. Acesso: 16 abr. 2020 .

Saha, S., Moortrhi, S., Pan, H. L., Wu, X., Wang, J., Nadiga, S., Tripp, P., Behringer, D., Hou, Y. T., Chuang, H.Y., Iredell, M., Ek, M., Meng, J., Yang, R., Mendez, M. P., Dool, H., Zhang, Q., Wang, W., Chen, M., Becker, E., 2014. The NCEP Climate Forecast System Version 2. Journal of Climate [Online] 27. Disponível: https://doi.org/10.1175/JCLI-D12-00823.1. Acesso: 16 abr. 2020.

Sanders, F., Gyakum, J.R., 1980. Synopticdynamic climatology of the "Bomb". Monthly Weather Review [Online] 108. Disponível: https://doi.org/10.1175/1520- 
0493(1980)108<1589:SDCOT>2.0.CO;2.

Acesso: 16 abr. 2020.

Santurette, P., Georgiev, C.G., 2005. Weather analysis and forecasting: Applying satellite water vapor imagery and potential vorticity analysis, 1 ed. Academic Press, Amsterdam.

Satyamurty, P., Ferreira, C. C., Gan, M. A., 1990. Cyclonic vortices over South America. Tellus A 42A, 194-201.

Schultz, D. M., Bosart, L. F., Colle, B. A., Davies, H. C., Dearden, C., Keuser, D., Martius, O., Roebber, P. J., Steenburgh, W. J., Volkert, H., Winters, A., 2018. Extratropical Cyclones: A Century of Research on Meteorology's Centerpiece. Meteorological Monographs [Online] 59.5 Disponível: https://doi.org/10.1175/AMSMONOGRAPH S-D-18-0015.1. Acesso: 16 abr. 2020.

Sinclair, M.R., 1994. An objective cyclone climatology for the Southern Hemisphere. Monthly Weather Review 122, 2239-2256.
Seluchi, M. E., Saulo, A. C., Nicolini, M., Satyamurty, P., 2003. The Northwestern Argentinean Low: A study of two typical events. Monthly Weather Review [Online] 131. Disponível:

https://doi.org/10.1175/15200493(2003)131<2361:TNALAS>2.0.CO;2. Acesso: 16 abr. 2020.

Taljaard, J. J., 1967. Development, distribution and movement of cyclones and anticyclones in the Southern Hemisphere during the IGY. Journal of Applied Meteorology 6, 973-987.

Vaughan, G., Price, J.D., Howells, A., 1994. Transport into the troposphere in a tropopause fold. Quarterly Journal of the Royal Meteorological Society [Online] 120. Disponível: https://doi.org/10.1002/qj.49712051814. Acesso: 16 abr. 2020. 